\title{
Addressing suicide risk in partners and relatives bereaved by suicide
}

Alexandra Pitman

\section{Summary}

Analyses of population-based registers provide evidence to support an increased risk of suicide and mental illness after the suicide of a relative or partner. This editorial explores the implications of this work for progressing recommendations made in suicide prevention strategies regarding the specific support provided to people bereaved by suicide.

\section{Declaration of interest}

A.P. served on the Public Health England expert reference committee contributing to local guidance on commissioning and delivering postvention support (Public Health England, 2016. Support After a Suicide: A Guide to Providing Local Services) and has submitted applications for funding to investigate mediators of suicide risk after suicide bereavement. Since writing this article she has become a Patron of the Support After Suicide Partnership.

\section{Copyright and usage}

(c) The Royal College of Psychiatrists 2018.
Alexandra Pitman is a senior clinical lecturer in the UCL Division of Psychiatry and Consultant Psychiatrist in the Camden and Islington NHS Foundation Trust. Her MRC doctoral fellowship investigated the association between bereavement by suicide and risk of suicide attempt.
Although a family history of suicide is an established risk factor for suicide, research and clinical attention has tended to focus on its genetic component. ${ }^{1}$ Consequently, mental health professionals routinely screen for a family history of suicide, but few are prompted to explore its emotional impact on the patient.

The environmental contribution of suicide exposure is now better understood thanks to studies linking the longitudinal routine health records of relatives and partners. This populationbased approach accesses large samples, and overcomes the selection, response and recall biases inherent to survey designs. From this work it is clear that beyond genetic risk, ${ }^{1}$ suicide bereavement confers an environmental contribution to suicide risk in unrelated partners, ${ }^{2,3}$ particularly men. ${ }^{2}$

Studies of blood-related individuals using Dutch and Danish registries also hint at environmental contributions. Children of mothers who die by suicide have a more marked risk of suicide than the children of fathers dying by suicide, particularly for mothers under 40 years of age. ${ }^{1}$ Among parents bereaved by a child's suicide, the magnitude of risk is greater in mothers than fathers. ${ }^{4}$ Although genetic expressivity is one explanation, these gender differences have been interpreted as reflecting differing bereavement reactions, ${ }^{1,4}$ pointing to a dual genetic and environmental effect. Together this evidence presents a strong case for policymakers to support the commissioning of services for the suicidebereaved, with the objective of preventing suicide closely allied to that of responding to distress.

\section{Policy responses}

The elevated risk of suicide after the suicide of a spouse was identified over a decade ago, ${ }^{2}$ but limited progress has been made in addressing it. The suicide prevention strategies of most highincome countries advise comprehensive support programmes for people bereaved by suicide, also known as postvention, but are vague on their components. The dearth of trial evidence is to blame. A 2008 systematic review in this Journal highlighted the lack of interventions found to reduce suicide risk in people bereaved by suicide, and the few shown to improve their mental health or social functioning. ${ }^{5}$ Whereas accumulating research studies support a range of interventions to address pathological grief in people bereaved by any cause, ${ }^{6}$ applying broad guidelines based on evidence from heterogeneous populations ignores issues specific to suicide loss. Approaches that fail to acknowledge the shame and self-stigma that tend to occur after a suicide ${ }^{3}$ are unlikely to be acceptable. Interventions that fail to address the specific mediators of suicide risk are unlikely to mitigate this risk. As yet, no epidemiological studies have identified the factors mediating suicide risk in people bereaved by suicide. Trial activity has stalled and needs invigorating. Gaining impetus relies on developing interventions addressing specific mediators of suicide risk while also meeting the need for support.

\section{Likely pathways}

Pathways to suicide in people bereaved by suicide can be hypothesised from available evidence. A recent Danish study of adults bereaved by a partner's suicide identified specific psychiatric disorders associated with suicide bereavement ${ }^{3}$ that are likely contributors to suicide risk. Suicide-bereaved partners, defined by marriage, civil union (legal in Denmark since 1989) or cohabitation, were at increased risk of incident mental disorder (specifically mood disorders and post-traumatic stress disorder). ${ }^{3}$ They had higher rates of psychiatric admission after the death, and an increased risk of suicide and all-cause mortality. ${ }^{3}$ Unlike suicide-bereaved men, women were at increased risk of self-harm and homicide. ${ }^{3}$ Although rates of substance misuse exceeded those for the general population, they were similar to those for bereaved controls. ${ }^{3}$

A Canadian longitudinal study of parents bereaved by suicide identified a substantial burden of mental and physical health problems even before their loss. ${ }^{7}$ Likely explanations include genetic traits shared with offspring for suicidality and mental illness, assortative mating based on the same traits, shared social adversity and the strain of being on suicide watch. These findings suggest a need to design acceptable systems for screening and treating incident or worsening psychiatric illness in partners and relatives bereaved by suicide, delivered in a manner that avoids pathologising grief. 
Despite these vulnerabilities, survey evidence shows that the suicide-bereaved are poorly supported by family and friends and may feel too ashamed or unworthy to ask for support. ${ }^{8}$ The influence of stigma on help-seeking and on others' propensity to offer help may also contribute to suicide risk. In a British survey, people bereaved by suicide were less likely to report receiving informal support than bereaved controls, or to have received formal or informal support in the immediate aftermath. ${ }^{8}$ Differences became non-significant when adjusting for perceived stigma. ${ }^{8}$

Danish men bereaved by a partner's suicide were less likely to consult their general practitioner than bereaved controls, despite their greater likelihood of mental illness. ${ }^{3}$ These men were no more likely than the general population to be prescribed antidepressants, ${ }^{3}$ albeit in a society with generally high rates of antidepressant prescribing. Bereaved at a mean age of 54, they had lost a key confidant(e) and the main social influence on their healthcare helpseeking. Their help-seeking behaviour might explain the much higher magnitude of suicide risk in bereaved male than female partners. ${ }^{2}$ Together this evidence suggests that systems of proactive outreach are indicated after suicide so that access to support does not rely on bereaved individuals self-referring.

Studies describing the timing of onset of psychiatric illness and suicidality are critical in identifying windows of preventive opportunity. After a child's death, parents' risk of suicide is particularly marked in the first month. ${ }^{4}$ These early weeks are a period during which people bereaved by suicide report being particularly poorly supported, ${ }^{8}$ reinforcing their sense of shame and self-stigma. Early intervention during this vulnerable period is likely to be indicated given the potential for deteriorating mental illness to contribute to suicide risk. This also promotes help-seeking for psychosocial difficulties by countering early on the stigmatising experience of being avoided by one's own network. ${ }^{8}$

\section{Service evaluation}

Services provided after suicide bereavement have been largely driven by the voluntary sector, responding to the demand for individual bereavement counselling and group peer support. Geographical provision is variable, particularly in rural areas, where suicide rates may be higher. Survey evidence indicates that a quarter of British people bereaved by suicide receive no formal or informal support after the loss, as distinct from those who prefer to handle it alone. ${ }^{8}$ A 2017 revision of the English suicide prevention strategy acknowledged poor progress in supporting families bereaved by suicide, ${ }^{9}$ and pledged to strengthen its focus by issuing local commissioning guidance. This emphasises collaborations between first responders (often emergency services), coroners' officers, the voluntary sector and healthcare services, such as the systems of early proactive outreach currently being piloted in the $\mathrm{UK}^{9}$ and elsewhere. Local service evaluations provide valuable information on acceptability and uptake, identifying whether early intervention is likely to be welcomed, but we also need trial evidence of effectiveness to demonstrate the clinical impact and maximise the sustainability of funding.

With so much attention currently focused on commissioning services for the suicide-bereaved, there is a need to fill evidence gaps swiftly to inform implementation. Priorities include identifying the mediators of suicide risk in partners and relatives bereaved by suicide, including exploring the role of suicide suggestion (or contagion), and identifying acceptable screening systems to address incident or deteriorating mental illness. These are necessary first steps before designing controlled trials to assess whether services are effective in reducing suicide attempt rates. Assessments of their effects on proximal outcomes, such as mental illness, help-seeking behaviour, pathological grief and social functioning, are also important, as is measurement of potential adverse effects. ${ }^{10}$ The results of these studies can be used to inform commissioning of services for suicide-bereaved partners, relatives and friends: a group prioritised within suicide-prevention strategies but ostracised by those in their social networks. ${ }^{8}$ Suicide prevention interventions are notoriously hard to evaluate, but only with trial evidence of clinical and cost-effectiveness in addressing the observed risks of mental illness ${ }^{3}$ and suicide ${ }^{2,3}$ can we be more directive with the recommendations made in suicide prevention policies, and more certain that they might have their intended effect.

Alexandra Pitman, BA MSc(Econ) MRCPsych PhD, UCL Division of Psychiatry, University College London, UK

Correspondence: Alexandra Pitman, Division of Psychiatry, University College London, Maple House, 149 Tottenham Court Road, London W1T 7NF. Email: a.pitman@ucl.ac.uk First received 19 Jun 2017, final revision 6 Oct 2017, accepted 19 Nov 2017

\section{References}

1 Garssen J, Deerenberg I, Mackenbach JP, Kerkhof A, Kunst AE. Familial risk of early suicide: variations by age and sex of children and parents. Suicide Life Threat Behav 2011; 41: 585-93.

2 Agerbo E. Midlife suicide risk, partner's psychiatric illness, spouse and child bereavement by suicide or other modes of death: a gender specific study. J Epidemiol Community Health 2005; 59: 407-12.

3 Erlangsen A, Runeson B, Bolton JM, Wilcox HC, Forman JL, Krogh J, et al. Association between spousal suicide and mental, physical, and social health outcomes: a longitudinal and nationwide register-based study. JAMA Psychiatry 2017; 74: 456-64.

4 Qin P, Mortensen P. The impact of parental status on the risk of completed suicide. Arch Gen Psychiatry 2003; 60: 797-802.

5 McDaid C, Trowman R, Golder S, Hawton K, Sowden A. Interventions for people bereaved through suicide: systematic review. Br J Psychiatry 2008; 193: 438-43.

6 Boelen PA, Smid GE. Disturbed grief: prolonged grief disorder and persistent complex bereavement disorder. BMJ 2017; 357: j2016.

7 Bolton JM, Au W, Leslie WD, Martens PJ, Enns MW, Roos LL, et al. Parents bereaved by offspring suicide: a population-based longitudinal case-control study. JAMA Psychiatry 2013; 70: 158-67.

8 Pitman AL, Rantell K, Moran P, Sireling L, Marston L, King M, et al. Support received after bereavement by suicide and other sudden deaths: a cross-sectional UK study of 3432 young bereaved adults. BMJ Open 2017; 7: e014487.

9 Department of Health Preventing Suicide in England: Third Progress Report of the Cross-Government Outcomes Strategy to Save Lives. Department of Health, 2017.

10 de Groot M, Kollen BJ. Course of bereavement over 8-10 years in first degree relatives and spouses of people who committed suicide: Iongitudinal community based cohort study. BMJ 2013; 347: f5519. 